\title{
Two-dimension display and attribute marking of Hainan Tourism Geographic Information based on the WebGIS
}

\author{
Yili Shi \\ Hainan Normal University \\ Haikou, Hainan \\ e-mail: shiyili@hainnu.edu.cn
}

\author{
Chengyi Zhang \\ Hainan Normal University \\ Haikou, Hainan \\ e-mail: chengyizh@hainnu.edu.cn
}

\begin{abstract}
The geographic tourism system based on Web GIS is of great significance for the development of tourism. This paper discusses the basic structure and management of ArcGIS Server 10.0 as well as its development methods. We design and build the tourism geographic information system of Hainan based on ArcGIS Server, and it can realize map browsing, zoom in/out, moving, information query and mark for travel map, tourist attractions, routing of travel on the electronic map with the Internet. Travelers can arrange their own travel plans reasonably on an interactive interface.
\end{abstract}

\section{Keywords-Web GIS; Tourism Geographic Information System; attribute marking}

\section{INTRODUCTION}

The construction of Hainan International Tourism Island has become the significant national development strategy, and Hainan will be a world-class scenic resort by 2020 . Tourism will be a key industry to lead the economic development of Hainan. Data shows that the number of the overnight visitors in Hainan has increased from 15.16 million in 2005 to 33.2 million in 2012, and the tourism revenue has increased from 12.5 billion yuan in 2005 to 37.9 billion yuan in 2012. This paper tries to create the Hainan tourism geographic information system based on Web GIS,and its purpose is to provide a more comprehensive, detailed information services for tourists through the Internet about tourist attraction and the related service facilities. The system uses the a unique GIS spatial data management capabilities, integrating a lot of space and space travel data in an electronic travel map, so that visitors can browse, query, and retrieve relevant travel information, and the system has a strong spatial analysis functions as well. Visitors can be provided with buffer analysis, and shortest path analysis, In addition, the system provides spatial decision support services for tourists in accordance with their own standards and personal preferences.[1]

\section{SySTEM DEVELOPMENT PlatFormS}

ArcGIS is a comprehensive system that allows people to collect, organize, manage, analyze, communicate, and distribute geographic information [2]. This article develops Web Gis by selecting ArcGIS Server 10.0 as a geographical information publishing platform, taking Hainan tourism map as the publishing object, with .Net Framework technology, and achieves the map browsing, attribute query, and other basic functions. By using the classic multilayer software architecture [3], Web Gis not only logically divides the function of each module and the relationship of each other, but also realize the physical implementation client applications, Web Server Extensions, GIS application servers, and data servers' independent real components. Each component can be used alone to maintain upgrade and update, the Web GIS Architecture is shown in Fig. 1:

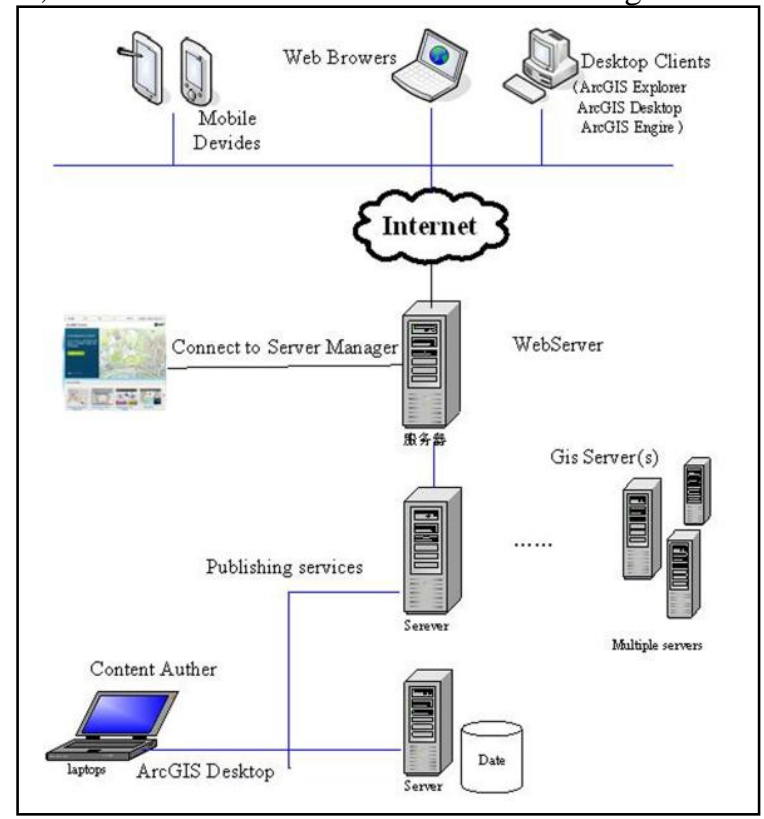

Figure 1. Web GIS Architecture (Four-layer construction mode)

Taking into account of the application of security and stability of the system, we select Win7 as the computer operating system, selecting third-generation Internet platform Microsoft Visualc\#.net as development language, the back-end database using Microsoft SQL Server 2005.

\section{THE DESIGN OF HAINAN TOURISM GEOGRAPHIC INFORMATION SYSTEM}

\section{A. Design Principles [4]}

- Clear goals;

- Advanced: adopt the currently popular development technologies and platforms;

- Scalability: The system uses modular structure, module independence each other, the system is easy to improve and expand;

- Intelligence: be able to make intelligent decisions based on system knowledge and database data; 
- Safety: The system should ensure the data is real and can not be tampered with

- Friendly interface: The system interface is simple and elegant, easy to operate.

\section{B. The Design Of The Attribute Of Hainan Tourism Attractions}

All the tourist attractions of Hainan are put in a separate layer, each of the spots is a feather of a layer. Attractions can be classified according to their characteristics, it is necessary to add the corresponding fields in the layer, For example, names of scenic spots, red tourism attractions, and levels etc., the attributes of the hainnan tourism attractions are shown below:

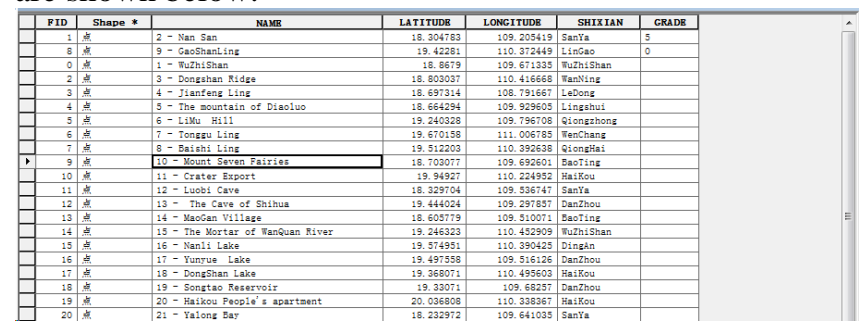

Figure 2. the attributes of the hainnan tourism attractions

\section{The Design Of System Functions}

The system should include the following three aspects According to the design objectives and design principles:

- (1) Basic information query

- (2) GIS functions

GIS functions includes(1)layer loading and display of the main attractions, major cities, administrative areas, boundaries, and land; (2)Map window zoom in, zoom out, recovery and other basic operations; (3)Hawkeye is a thumbnail map, it can realize full map navigation when move the rectangular in Hawkeye; (4)Search the Attractions attribute information from property table; (5)The attribute selected from map can be located and highlighted [5].

- (3) The functions of intelligent decision support

Decision support module provides intelligent decision support services to tourists according to the user's personal preference tendency as well as tourists travel conditions (time, travel costs).

\section{The Design Of Overall Structure}

According to the design objectives and design principles of the system, as well as functional requirements [6], the overall structure of the system design shown in Fig. 3:

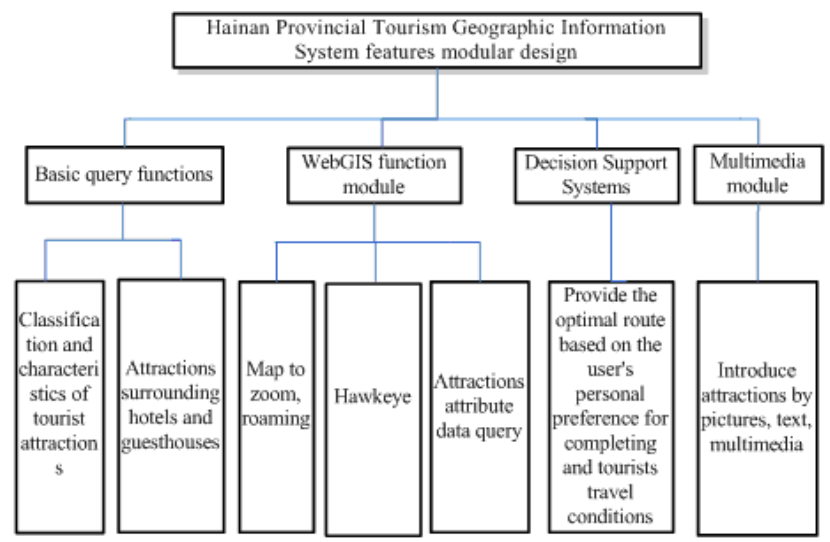

Figure 3. The overall structure of the system

\section{THE IMPLEMENTATION OF HAINAN TOURISM GEOGRAPHIC INFORMATION SYSTEM}

This paper realizes the release of Hainan tourism geographic information through the Internet after researching the overall framework of Web GIS and related technology. The visitors can query Geographic Information and intelligently plan their own travel route based on Hainan tourism geographic information platform, and the results of intelligent analysis can be hierarchically displayed and with attribute making.

\section{A. The Design Of The Main Interface In Travel Map System}

According to the design goals and functional requirements of the system, the main operation interface is shown in Fig. 4, including the map display area, operating tools area, Layer control area, search area, the resulting output area, and so on. The Main interface of Hainan tourism geographic information system is shown in Fig. 4:

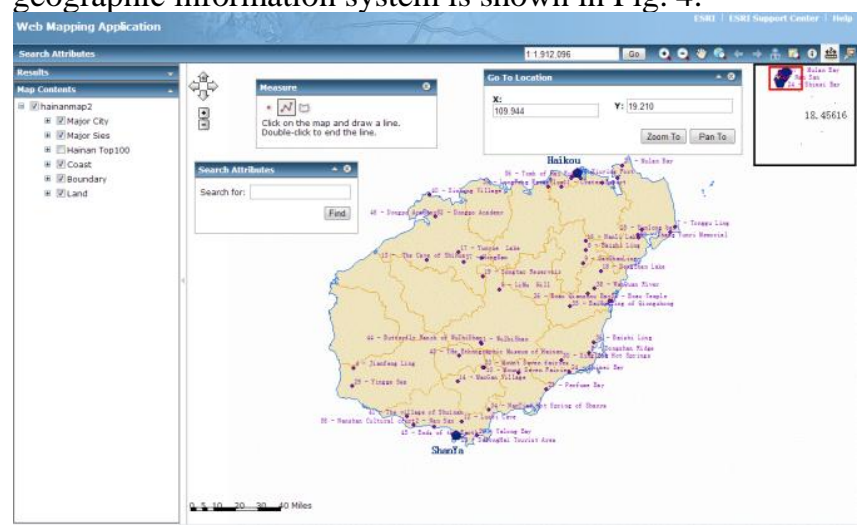

Figure 4. Main interface of Hainan tourism geographic information system

\section{B. The Major Steps To Release Travel Map}

- ArcGIS Desktop completes the production of the Hainan Provincial Tourism Map.

ArcGIS Desktop could create and handle different types of Hainan Geographic tourism information.

- Publishing services. 
The ArcGIS Server 10 releases map information in this paper; A GIS server hosts GIS services. A GIS service represents a GIS resource, such as a map, geographic database that is located on the server and is made available for clients applications.

- Creating Web applications

We can use ArcGIS Server Manager to create and edit WebGIS client application applications that show the tourism geographic information running on your server, user can obtain the Tourism geographic information through the Internet.

\section{The Function Module Of GIS.}

The GIS module includes Zoom in, zoom out, roaming, pan, attribute query, measure, map management editing, and so on. Administrators can locally or remotely manage the entire system, including the choice with the basic settings of the map, spatial data and attribute data editing and maintenance, which can achieve real-time updates to the system to provide real-time information services for tourists.

- (1) The classification query of Attractions by attributes

Results of Search attractions by location attributes (e.g.: Sanya) are shown in Fig. 5:

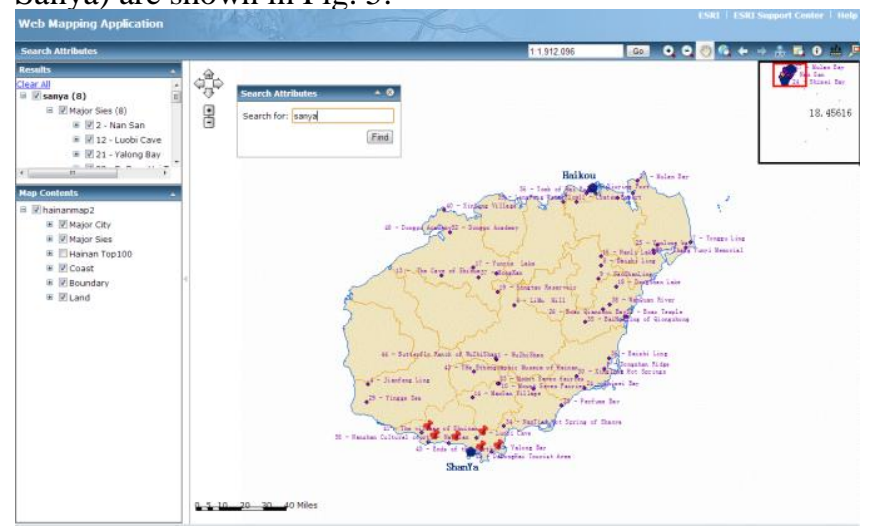

Figure 5. The attribute query results by location (Sanya)

\section{Decision Support Function}

We can provide the best route of travel, distance, time consuming, cost and other information which are two-dimension display and attribute marking, according to the user's personal preference tendency. Fig. 6 shows the route map, related costs and attribute marking of the four 5A class scenic spots if the tourist starts from Sanya. The four $5 \mathrm{~A}$ scenic route map, related costs and attribute label are shown in Fig. 6:

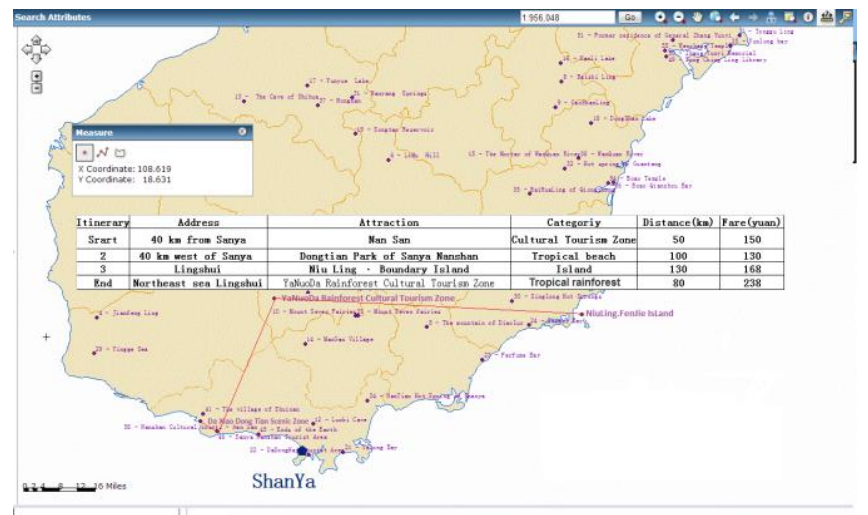

Figure 6. The four 5A scenic route map, related costs and attribute label

\section{CONCLUSIONS}

In this paper, we achieve a Web GIS system based on the ArcGIS Server platform by using the Hainan Tourism Geographic Information as published objects. The system can offer tourism geographic Information services for tourist, including map browsing, query attributes, decision support and other basic functions, and are of great significance in promoting the development of tourism information industry in Hainan.

\section{ACKNOWLEDGMENT}

This work is supported by NSF of China (71140008)and Hainan special social development technology (2011SFO3). Corresponding author: Chengyi Zhang. E-mail: Chengyizh@hainnu.edu.cn.

\section{REFERENCES}

[1] Chen Zan-zhang,Analysis and Design of the Hainan Tourism Geography Information System Based on WebGIS:GEOSPATIAL INFORMATION,Aug.,2012,Vol.10,No.4 ,pp.49

[2] http://help.arcgis.com/en/arcgisserver/10.0/help/arcgis_server_dotnet _help/index.html\#//00930000000n000000,2012

[3] LIU Xiao-hui, LIU Yong-wei, LIU Yong, Research on the distributed architecture of ArcGIS Server and strategy of server deployment: Science of Surveying and Mapping(j), 2011,7,pp.236

[4] Li Zhan-cheng Design and Realization of Tourism Geographic Information System Using on Web GIS: JOURNAL OF EAST CHINA INSTITUTE OF TECHNOLOGY, 2007.3,pp.68-73

[5] Sun Lei,Li Xin-ying, Based on the WebGIS of Sanya Tourism Geographic Information System Design and Development: Science \& Technology Vision(j), 2012,7,pp.10

[6] Cheng zhu,Reseach and Development of WebGIS based on .NET and ArcGIS Server(m), 2010.4,pp.31 